Pacific Journal of Mathematics

ON VALUE DISTRIBUTION OF FUNCTIONS MEROMORPHIC 


\title{
ON VALUE DISTRIBUTION OF FUNCTIONS MEROMORPHIC IN THE WHOLE PLANE
}

\author{
Hidenobu Yoshida
}

\begin{abstract}
We make two kinds of decompositions of functions meromorphic in the whole plane into two classes: One is the class of meromorphic functions of the first kind in the sense of Yosida (resp. of the first kind in the sense of Gavrilov) and the other is the class of meromorphic functions of the second kind in the sense of Yosida (resp. of the second kind in the sense of Gavrilov). Using these decompositions, we prove a result about the growth of the characteristic functions and some results about value distribution, of meromorphic functions of the first kind in the sense of Yosida (resp. of the first kind in the sense of Gavrilov).
\end{abstract}

1. Introduction. Noshiro [6] decomposed the class of normal meromorphic functions in the unit disc into two categories: Normal meromorphic functions of the first category and those of the second category. Then, Tse [8] generalized this idea to general meromorphic functions in the unit disc and decomposed all functions meromorphic in the unit disc into two classes: One is the class of meromorphic functions of the first kind and the other is the class of meromorphic functions of the second kind. Using this decomposition, he proved some interesting results about value distibution of meromorphic functions in the unit disc.

On the other hand, Yosida [10] defined a class of meromorphic functions in the whole plane, called it the class (A) and divided this class into two categories: Meromorphic functions of the first category and those of the second category. Then, Gavrilov [2] divided the class $W_{1}$ of Julia's exceptional functions into two subclasses: The class $W_{1}^{0}$ and the class of functions not belonging to $W_{1}^{0}$. Using each of these notions, they proved some interesting results.

In this paper, by making a consideration parallel to Tse's, we generalize both Yosida's decomposition and Gavrilov's decomposition to general meromorphic functions in the whole plane and according to each generalization we make two kinds of decompositions of functions meromorphic in the whole plane into two classes. Using these decompositions, we prove some results about value distribution of meromorphic functions in the whole plane.

I wish to express my thanks to the referee of this paper for his kind remarks. 
2. Preliminaries. We denote the whole $z$-plane by $Z$ and the $w$-sphere by $W$. Let $z_{0}$ be a point in $Z$ and $\epsilon$ be a positive number. By $D\left(z_{0}, \epsilon\right)$ (resp. $D^{*}\left(z_{0}, \epsilon\right)$, where $z_{0} \neq 0$ ), we denote the set

$$
\left.\left\{z ;\left|z-z_{0}\right|<\epsilon\right\} \quad \text { (resp. }\left\{z ;\left|z-z_{0}\right|<\epsilon \cdot\left|z_{0}\right|\right\}\right) .
$$

A meromorphic function $f(z)$ in $Z$ is said to be of the second kind in the sense of Yosida (resp. of the second kind in the sense of Gavrilov), if there exists a sequence $\left\{z_{n}\right\}$ of points in $Z,\left|z_{n}\right| \rightarrow \infty$, such that the sequence $\left\{f_{n}(z)\right\}$ of functions $f_{n}(z)=f\left(z+z_{n}\right)$ (resp. $f_{n}(z)=$ $\left.f\left(\left|z_{n}\right| \cdot z+z_{n}\right)\right)$, tends uniformly to a constant on $W$ in some neighborhood of $z=0$. A meromorphic function $f(z)$ in $Z$ is said to be of the first kind in the sense of Yosida (resp. of the first kind in the sense of Gavrilov), if it is not of the second kind in the sense of Yosida (resp. of the second kind in the sense of Gavrilov).

REMARK 2.1. A meromorphic function $f(z)$ in $Z$ is of the second kind in the sense of Yosida (resp. of the second kind in the sense of Gavrilov) if, and only if there exist a sequence $\left\{z_{n}\right\}$ of points in $Z$, $\left|z_{n}\right| \rightarrow \infty$, and a positive constant $\epsilon$. such that $f(z)$ tends uniformly to a constant on the set

$$
\bigcup_{n} D\left(z_{n}, \epsilon\right) \quad\left(\text { resp. } \bigcup_{n} D^{*}\left(z_{n}, \epsilon\right)\right)
$$

as $|z| \rightarrow \infty$. From this fact, we can speak roughly: Meromorphic functions of the second kind in the sense of Yosida (resp. of the second kind in the sense of Gavrilov) behave mildly, while those of the first kind in the sense of Yosida (resp. of the first kind in the sense of Gavrilov) are comparatively wild in the sense that the values which they assume do not tend to any constant limit on sequences of discs $D(z, \epsilon)\left(\right.$ resp. $\left.D^{*}(z, \epsilon)\right)$ for any fixed positive number $\epsilon$.

Remark 2.2. As "the class (A)", Yosida [10] called the class of meromorphic functions having the following property: For any sequence $\left\{z_{n}\right\}$ of complex numbers, the family $\left\{f_{n}(z)\right\}$ of meromorphic functions $f_{n}(z)=f\left(z+z_{n}\right)$ is a normal family in $Z$. And he divided this class into two categories: $f(z)$ is a function of the first category if there is no sequence $\left\{z_{n}\right\}$ of complex numbers such that the normal family $\left\{f_{n}(z)\right\}$ admits a constant limit, and any function which is not of the first category is of the second category. It is evident that a meromorphic function belonging to the class (A) is of the second category if and only if it is of the second kind in the sense of Yosida.

According to Gavrilov [2], a Julia's exceptional function $f(z)$ 
belongs to $W_{1}^{0}$ if there is no sequence $\left\{z_{n}\right\}$ of points in $Z,\left|z_{n}\right| \rightarrow \infty$, such that the normal family $\left\{g_{n}(z)\right\}, g_{n}(z)=f\left(z_{n} \cdot z\right)$, in $\{z ; 0<|z|<\infty\}$, admits a constant limit. Now, if we consider the sequence $\left\{h_{n}(t)\right\}$, $h_{n}(t)=f\left(z_{n}(1+t)\right)=f\left(z_{n}+z_{n} \cdot t\right)$, in a neighborhood of $t=0$, which is the same as $\left\{g_{n}(z)\right\}$ in a neighborhood of 1 , it is evident that a Julia's exceptional function belongs to $W_{1}^{0}$ if and only if it is a meromorphic function of the first kind in the sense of Gavrilov.

REMARK 2.3. There exists an interesting example of integral functions of the first kind in the sense of Yosida (resp. in the sense of Gavrilov). From Yosida [10, Theroem 6] (resp. Lehto and Virtanen [3, p. 7-8]), we see that this function does not belong to the class (A) (resp. the class of Julia's exceptional functions).

So, in order to obtain this example, we shall define the following terms, in the manner analogous to the case of the unit disc. Let $\zeta(t)$ be a continuous, complex-valued function for $0 \leqq t<\infty$ with the properties:

$$
\lim _{t \rightarrow \infty}|\zeta(t)|=+\infty, \quad \lim _{t \rightarrow \infty} \arg \zeta(t)=+\infty
$$

and let the equation $z=\zeta(t)$ define a simple curve. We denote by $S$ the set $\{z ; z=\zeta(t), 0 \leqq t<\infty\}$ and we call it a spiral in $Z$. Then, from Mergelyan [4, Theorem 1.2., p. 326], we see that $S$ is a Carleman continuum, and hence, we can find an integral function $f(z)$ such that $f(z)$ is bounded on $S$. We shall refer to integral functions with the property that it remains bounded on some spiral $S$ in $Z$, as integral functions of class $(V)$ relative to $S$ in $Z$. We shall also introduce the following measures for the "tightness" of a spiral in $Z$ whose equation is $z=\zeta(t)$. For any value of $t$, starting with the point $\zeta(t)$, describe the curve in the sense of increasing $t$ and let $t^{\prime}$ denote the first value of $t$ for which $\arg \zeta\left(t^{\prime}\right)=\arg \zeta(t)+2 \pi$. Then, we put

$$
\bar{\mu}(S)=\varlimsup_{t \rightarrow \infty}\left|\zeta(t)-\zeta\left(t^{\prime}\right)\right|, \quad \bar{\mu}^{*}(S)=\varlimsup_{t \rightarrow \infty} \frac{\left|\zeta(t)-\zeta\left(t^{\prime}\right)\right|}{|\zeta(t)|}
$$

Now, take an integral function $f(z)$ of class (V) relative to a spiral $S$ in $Z$, for which $\bar{\mu}(S)=0$ (resp. $\bar{\mu}^{*}(S)=0$ ). Then, since any integral function has an asymptotic value $\infty$, there is no sequence $\left\{z_{n}\right\}$ of points in $Z, \quad\left|z_{n}\right| \rightarrow \infty$, such that the sequence $f_{n}(z)=f\left(z+z_{n}\right)$ (resp. $\left.f_{n}(z)=f\left(\left|z_{n}\right| \cdot z+z_{n}\right)\right)$, for this $f(z)$, converges uniformly to a constant in a neighborhood of $z=0$. Therefore, this $f(z)$ is of the first kind in the sense of Yosida (resp. of the first kind in the sense of Gavrilov). 
3. The characteristic functions of meromorphic functions of the first kind. First of all, we shall prove a characteristic property of meromorphic functions of the first kind in the sense of Yosida (resp. of the first kind in the sense of Gavrilov) (Theorem 1).

Lemma 1. (See Ostrowski [7, Satz 1 and p. 234].) Let $\left\{z_{n}\right\}$ be a sequence of points in $Z$ satisfying $\left|z_{n}\right| \rightarrow \infty$ and $f(z)$ be a meromorphic function in $Z$. Then, if the family $\left\{f_{n}(z)\right\}$ of functions

$$
f_{n}(z)=f\left(z+z_{n}\right) \quad\left(\text { resp. } f_{n}(z)=f\left(\left|z_{n}\right| \cdot z+z_{n}\right)\right)
$$

is not normal at $z=0$, we can choose a subfamily $\left\{f_{n_{k}}(z)\right\}$ of $\left\{f_{n}(z)\right\}$ such that for any $\epsilon, \epsilon>0$, and any $\delta, \delta>0$, each of functions $f_{n k}(z)$ for $k>k_{0}$ assumes every values on $W$ in $D(0, \epsilon)$ with the possible exception of two sets of values $E$ and $G$ on $W$ whose spherical diameters do not exceed $\delta$, where $k_{0}$ is a positive integer dependent on $\epsilon$ and $\delta$, and both $E$ and $G$ are dependent on $\epsilon, \delta$ and $k$.

THEOREM 1. In order that a meromorphic function $f(z)$ in $Z$ is of the first kind in the sense of Yosida (resp. of the first kind in the sense of Gavrilov), it is necessary and sufficient that for each positive number $\epsilon$

$$
\begin{gathered}
\underset{\left|z_{0}\right|<\infty}{\operatorname{infimum}} \iint_{D\left(z_{0}, \epsilon\right)}\left(\frac{\left|f^{\prime}(z)\right|}{1+|f(z)|^{2}}\right)^{2} d \sigma_{z}>0 \\
\text { (resp. } \left.\underset{1 \leqq\left|z_{0}\right|<\infty}{\operatorname{infimum}} \iint_{D^{*}\left(z_{0}, \epsilon\right)}\left(\frac{\left|f^{\prime}(z)\right|}{1+|f(z)|^{2}}\right)^{2} d \sigma_{z}>0\right),
\end{gathered}
$$

where $d \sigma_{z}$ denotes the area element.

Proof. The condition is sufficient. Suppose that there exist a sequence $\left\{z_{n}\right\}$ of points, $\left|z_{n}\right| \rightarrow \infty$ (if necessary, we may suppose $\left|z_{n}\right| \geqq 1$ ), and a neighborhood $D(0, \epsilon)$ such that the sequence

$$
f_{n}(z)=f\left(z+z_{n}\right) \quad\left(\text { resp. } f_{n}(z)=f\left(\left|z_{n}\right| \cdot z+z_{n}\right)\right)
$$

tends uniformly to a constant in $D(0, \epsilon)$. Then, we have that

$$
\begin{aligned}
& \iint_{D(0, \epsilon)}\left(\frac{\left|f_{n}^{\prime}(z)\right|}{1+\left|f_{n}(z)\right|^{2}}\right)^{2} d \sigma_{z}=\iint_{D\left(z_{n, \epsilon)}\right.}\left(\frac{\left|f^{\prime}(z)\right|}{1+|f(z)|^{2}}\right)^{2} d \sigma_{z} \rightarrow 0 \quad(n \rightarrow \infty) \\
& \text { resp. } \iint_{D(0, \epsilon)}\left(\frac{\left|f_{n}^{\prime}(z)\right|}{1+\left|f_{n}(z)\right|^{2}}\right)^{2} d \sigma_{z} \\
& \left.\quad=\iint_{D^{*}\left(z_{n}, \epsilon\right)}\left(\frac{\left|f^{\prime}(z)\right|}{1+|f(z)|^{2}}\right)^{2} d \sigma_{z} \rightarrow 0 \quad(n \rightarrow \infty)\right) .
\end{aligned}
$$


This fact contradicts (3.1).

The condition is necessary. Suppose that there exist a positive constant $\epsilon$ and a sequence $\left\{z_{n}\right\},\left|z_{n}\right| \rightarrow \infty$ (resp. $\left|z_{n}\right| \rightarrow \infty,\left|z_{n}\right| \geqq 1$ ), such that

$$
\iint_{D\left(z_{n, \epsilon)}\right.}\left(\frac{\left|f^{\prime}(z)\right|}{1+|f(z)|^{2}}\right)^{2} d \sigma_{z}=\iint_{D(0, \epsilon)}\left(\frac{\left|f_{n}^{\prime}(z)\right|}{1+\left|f_{n}(z)\right|^{2}}\right)^{2} d \sigma_{z} \rightarrow 0 \quad(n \rightarrow \infty)
$$

(resp. $\iint_{D^{*}\left(z_{n}, \epsilon\right)}\left(\frac{\left|f^{\prime}(z)\right|}{1+|f(z)|^{2}}\right)^{2} d \sigma_{z}$

$$
=\iint_{D(0, \epsilon)}\left(\frac{\left|f_{n}^{\prime}(z)\right|}{1+\left|f_{n}(z)\right|^{2}}\right)^{2} d \sigma_{z} \rightarrow 0 \quad(n \rightarrow \infty) .
$$

Then, the family $\left\{f_{n}(z)\right\}$,

$$
f_{n}(z)=f\left(z+z_{n}\right) \quad\left(\text { resp. } f_{n}(z)=f\left(\left|z_{n}\right| \cdot z+z_{n}\right)\right),
$$

for this sequence $\left\{z_{n}\right\}$ is normal at $z=0$. Because, if we suppose that this family $\left\{f_{n}(z)\right\}$ is not normal at $z=0$, we see from Lemma 1 that (3.2) can not hold. Thus, we can obtain a subfamily $\left\{f_{n_{k}}(z)\right\}$ of $\left\{f_{n}(z)\right\}$ and a neighborhood $D(0, \epsilon)$ such that $f_{n_{k}}(z)$ converges uniformly to a function $p(z)$ in $D(0, \epsilon)$. And we have that

$$
\iint_{D(0, \epsilon)}\left(\frac{\left|f_{n_{k}}^{\prime}(z)\right|}{1+\left|f_{n k}(z)\right|^{2}}\right)^{2} d \sigma_{z} \rightarrow \iint_{D(0, \epsilon)}\left(\frac{\left|p^{\prime}(z)\right|}{1+|p(z)|^{2}}\right)^{2} d \sigma_{z}
$$

as $n \rightarrow \infty$. Hence, we can conclude from (3.2) that

$$
\iint_{D(0, \epsilon)}\left(\frac{\left|p^{\prime}(z)\right|}{1+|p(z)|^{2}}\right)^{2} d \sigma_{z}=0
$$

But, on the other hand, since $f(z)$ is of the first kind in the sense of Yosida (resp. of the first kind in the sense of Gavrilov), we see that $p(z) \not \equiv$ constant, and hence,

$$
\iint_{D(0, \epsilon)}\left(\frac{\left|p^{\prime}(z)\right|}{1+|p(z)|^{2}}\right)^{2} d \sigma_{z}>0
$$

This contradicts (3.3).

Now, we can prove a result concerning the growth of the characteristic function $T(r, f)$ of $f(z)$ of the first kind in the sense of Yosida (resp. of the first kind in the sense of Gavrilov). 
THEOREM 2. Let $f(z)$ be a meromorphic function in $Z$ of the first kind in the sense of Yosida (resp. of the first kind in the sense of Gavrilov). Then, the characteristic function $T(r, f)$ of $f(z)$ has the property

$$
\varlimsup_{r \rightarrow \infty} \frac{T(r, f)}{r^{2}}>0 \quad\left(\text { resp. } \varlimsup_{r \rightarrow \infty} \frac{T(r, f)}{\log ^{2} r}>0\right) .
$$

Proof. From Theorem 1, for an $\epsilon, \epsilon>0$ (resp. $0<\epsilon<1$ ), we can find a positive number $\eta$ such that

$$
\iint_{D(z 0, \epsilon)}\left(\frac{\left|f^{\prime}(z)\right|}{1+|f(z)|^{2}}\right)^{2} d \sigma_{z}\left(\operatorname{resp} . \iint_{D^{*}(z 0, \epsilon)}\left(\frac{\left|f^{\prime}(z)\right|}{1+|f(z)|^{2}}\right)^{2} d \sigma_{z}\right) \geqq \eta
$$

for all $z_{0}$ (resp. for all $z_{0},\left|z_{0}\right| \geqq 1$ ). Using this fact, we shall estimate the evaluation of the characteristic function $T(r, f)$ of $f(z)$

$$
T(r, f)=\int_{0}^{r} \frac{S(r, f)}{r} d r, \quad S(r, f)=\frac{1}{\pi} \iint_{D(0, r)}\left(\frac{\left|f^{\prime}(z)\right|}{1+|f(z)|^{2}}\right)^{2} d \sigma_{z} .
$$

Take the sequence of real numbers $\left\{x_{n}\right\}$ decided by the following recurrence formula

$$
\begin{array}{r}
x_{1}=2 \epsilon, x_{n+1}=x_{n}+2 \epsilon\left(\text { resp. } x_{1}=1, x_{n+1}=x_{n}+\epsilon \cdot x_{n+1}+\epsilon \cdot x_{n}\right) \\
(n=1,2,3, \cdots)
\end{array}
$$

and consider the sequence of discs

$$
\Gamma_{n, 1}=D\left(x_{n}, \epsilon\right) \quad\left(\operatorname{resp} . \Gamma_{n, 1}=D^{*}\left(x_{n}, \epsilon\right)\right) \quad(n=1,2,3, \cdots) .
$$

Then, two discs $\Gamma_{n, 1}$ and $\Gamma_{n+1,1}$ intersect only at

$$
r_{n}=x_{n}+\epsilon \quad\left(\text { resp. } r_{n}=x_{n}+\epsilon \cdot x_{n}\right) \quad(n=1,2,3, \cdots) .
$$

Next, for the angle $\theta_{n}$ subtended at $z=0$ by the disc $\Gamma_{n, 1}$, we have

$$
\frac{\theta_{n}}{\pi}<\sin \left(\frac{\theta_{n}}{2}\right)=\frac{1}{2 n} \quad\left(\text { resp. } \frac{\theta_{n}}{\pi}<\sin \left(\frac{\theta_{n}}{2}\right)=\epsilon\right) \quad(n=1,2,3, \cdots) .
$$

Hence, in the annulus $\Omega_{n}=\left\{z ; r_{n-1}<|z|<r_{n}\right\}(n=1,2,3, \cdots)$, where $r_{0}=\epsilon$ (resp. $r_{0}=1-\epsilon$ ), we can arrange $4 n$ (resp. [2/ $\epsilon$ ], where [ ] denotes Gauss's symbol) number of discs $\Gamma_{n, l}(i=1,2,3, \cdots, 4 n)$ (resp. $i=1,2,3, \cdots,[2 / \epsilon])$, each of which has radius $\epsilon$ (resp. $\left.\epsilon \cdot x_{n}\right)$ and its center on $\left\{z ;|z|=x_{n}\right\}$, in the way that any two of these discs not intersect mutually. Thus, we have that for $n=1,2,3, \cdots$ 


$$
D\left(0, r_{n}\right) \supset \bigcup_{\substack{k=1,2,3, \cdots, n \\ l=1,2,3, \cdots, 4 k}} \Gamma_{k, i} \quad\left(\text { resp. } \bigcup_{\substack{k=1,2,3, \cdots, n \\ l=1,2,3, \cdots,[2 / \epsilon]}} \Gamma_{k, l}\right)
$$

Now, if we note (3.6) and the following fact obtained from (3.5):

$$
\begin{gathered}
\iint_{\Gamma_{n, t}}\left(\frac{\left|f^{\prime}(z)\right|}{1+|f(z)|^{2}}\right)^{2} d \sigma_{z} \geqq \eta \\
(n=1,2,3, \cdots ; i=1,2,3, \cdots, 4 n \text { (resp. } i=1,2,3, \cdots,[2 / \epsilon])),
\end{gathered}
$$

we can obtain the estimation

$$
\begin{aligned}
S\left(r_{n}, f\right) \geqq \frac{1}{\pi} \sum_{k=1}^{n} 4 \eta k(\operatorname{resp} . & \left.\frac{1}{\pi} \sum_{k=1}^{n}\left[\frac{2}{\epsilon}\right] \eta\right) \\
= & \frac{2 \eta}{\pi} n(n+1)\left(\operatorname{resp} \cdot \frac{\eta}{\pi}\left[\frac{2}{\epsilon}\right] n\right)(n=1,2,3, \cdots) .
\end{aligned}
$$

Hence, from

$$
r_{i}-r_{i-1}=2 \epsilon \quad\left(\text { resp. } 2 \epsilon\left(\frac{1+\epsilon}{1-\epsilon}\right)^{i-1}\right) \quad(i=1,2,3, \cdots)
$$

we have

$$
\begin{aligned}
& T\left(r_{n}, f\right) \geqq \sum_{i=2}^{n} \int_{r_{i-1}}^{r_{i}} \frac{S(r, f)}{r} d r \geqq \sum_{i=2}^{n} S\left(r_{i-1}, f\right) \frac{r_{i}-r_{i-1}}{r_{i}} \\
& \geqq \frac{2 \eta}{\pi} \sum_{i=2}^{n}(i-1)\left(\text { resp. } \frac{2 \epsilon}{1+\epsilon}\left[\frac{2}{\epsilon}\right] \frac{\eta}{\pi} \sum_{i=2}^{n}(i-1)\right) \\
&=A(n-1) n,
\end{aligned}
$$

where $A$ is a positive constant independent of $n$. Thus, from

$$
r_{n}=2 \epsilon n \quad\left(\text { resp. } r_{n}=(1+\epsilon)\left(\frac{1+\epsilon}{1-\epsilon}\right)^{n-1}\right)
$$

we obtain

$$
\begin{aligned}
\varlimsup_{n \rightarrow \infty} \frac{T\left(r_{n}, f\right)}{r_{n}^{2}} \geqq \frac{A n(n-1)}{4 \epsilon^{2} n^{2}} \geqq \frac{A}{4 \epsilon^{2}} \\
>0 \quad\left(\text { resp. } \varlimsup_{n \rightarrow \infty} \frac{T\left(r_{n}, f\right)}{\log ^{2} r_{n}} \geqq A / \log ^{2}\left(\frac{1+\epsilon}{1-\epsilon}\right)>0\right) .
\end{aligned}
$$

This proves Theorem 2 . 
REMARK 3.1. This Theorem 2 is sharp in the following sense: There exists a meromorphic function in $Z$ of the first kind in the sense of Yosida (resp. of the first kind in the sense of Gavrilov) whose characteristic function has the property

$$
0<\varlimsup_{r \rightarrow \infty} \frac{T(r, f)}{r^{2}}<\infty \quad\left(\text { resp. } 0<\varlimsup_{r \rightarrow \infty} \frac{T(r, f)}{\log ^{2} r}<\infty\right) .
$$

In fact, Yosida [10, 2. Corollary] (resp. Lehto and Virtanen [3, p. 9]) showed that the characteristic functions of meromorphic functions belonging to the class (A) of the first category (resp. of Julia's exceptional functions belonging to $W_{1}^{0}$ ) have the property (3.7).

REMARK 3.2. We remark that the inverse of Theorem 2 is not true. For example, consider the integral function

$$
\int_{0}^{z} e^{-t^{p}} d t
$$

where $p, p \geqq 3$, is a positive integer (see Nevanlinna [5, p. 170]).

REMARK 3.3. It is evident that a meromorphic function in $Z$ of the second kind in the sense of Gavrilov is of the second kind in the sense of Yosida. But, there exists a meromorphic function in $Z$ of the second kind in the sense of Yosida and of the first kind in the sense of Gavrilov. In fact, consider Julia's exceptional functions belonging to $W_{1}^{0}$. Then, we see from Remark 2.2 that these functions are of the first kind in the sense of Gavrilov. But since the characteristic functions of Julia's exceptional functions $f(z)$ have the property

$$
\varlimsup_{r \rightarrow \infty} \frac{T(r, f)}{\log ^{2} r}<\infty
$$

Theorem 2 shows that these functions are of the second kind in the sense of Yosida.

COROLLARY 1. Let $f(z)$ be a meromorphic function in $Z$ satisfying

$$
\varlimsup_{r \rightarrow \infty} \frac{T(r, f)}{r^{2}}=0 \quad\left(\text { resp. } \varlimsup_{r \rightarrow \infty} \frac{T(r, f)}{\log ^{2} r}=0\right) .
$$

Then, there exist a sequence $\left\{z_{n}\right\}$ of points, $\left|z_{n}\right| \rightarrow \infty$, and a positive constant $\epsilon$ such that $f(z)$ converges uniformly to a constant as $|z| \rightarrow \infty$, in the set 


$$
\bigcup_{n=1}^{\infty} D\left(z_{n}, \epsilon\right) \quad\left(\text { resp. } \bigcup_{n=1}^{\infty} D^{*}\left(z_{n}, \epsilon\right)\right)
$$

\section{The value distribution of meromorphic functions} in $Z$. Let $f(z)$ be a meromorphic function in $Z$. Gavrilov [1] defined the notion "a sequence of $M^{(0)}$-points for $f(z)$ ". Now, a sequence $\left\{z_{n}\right\}$ of points, $\left|z_{n}\right| \rightarrow \infty$, is called a sequence of pseudo-M $M^{(0)}$-points for $f(z)$ if for each increasing sequence $\left\{r_{n}\right\}$ of positive numbers, $r_{n} \uparrow \infty, f(z)$ assumes every value on $W$ infinitely often, with the possible exception of two values, in the set $\bigcup_{m=1}^{\infty} D\left(z_{m}, r_{m}\right)$ for any subsequence $\left\{z_{m}\right\}$ of $\left\{z_{n}\right\}$.

THEOREM 3. If $f(z)$ is a meromorphic function in $Z$ of the first kind in the sense of Yosida; then any sequence $\left\{z_{n}\right\}$ of points, $\left|z_{n}\right| \rightarrow \infty$, is a sequence of pseudo- $M^{(0)}$-points for $f(z)$.

Proof. Suppose that there exist a sequence $\left\{z_{n}\right\}_{n=1}^{\infty}$ of points, $\left|z_{n}\right| \rightarrow \infty$, a subsequence $\left\{z_{m}\right\}_{m=1}^{\infty}$ of $\left\{z_{n}\right\}_{n=1}^{\infty}$ and a sequence $\left\{r_{n}\right\}_{n=1}^{\infty}$ of positive numbers, $r_{n} \uparrow \infty$, such that $f(z)$ omits three values on $W$ in the union $\bigcup_{m=1}^{\infty} D\left(z_{m}, r_{m}\right)$. Then, since each function $f_{m}(z)=f\left(z_{m}+z\right)$ omits three values in $D\left(0, r_{1}\right)$, we can choose a subsequence $\left\{z_{i}^{(1)}\right\}_{i=1}^{\infty}$ of $\left\{z_{m}\right\}_{m=2}^{\infty}$ such that $f_{i}^{(1)}(z)=f\left(z_{i}^{(1)}+z\right)$ converges uniformly on each compact subset of $D\left(0, r_{1}\right)$. Next, since each function $f_{i}^{(1)}(z)$ omits three values in $D\left(0, r_{2}\right)$, we can choose a subsequence $\left\{z_{i}^{(2)}\right\}_{i=1}^{\infty}$ of $\left\{z_{i}^{(1)}\right\}_{i=2}^{\infty}$ such that $f_{i}^{(2)}(z)=f\left(z_{\imath}^{(2)}+z\right)$ converges uniformly on each compact subset of $D\left(0, r_{2}\right)$. In general, for each integer $p, p>0$, we can choose a subsequence $\left\{z_{i}^{(p+1)}\right\}_{i=1}^{\infty}$ of $\left\{z_{i}^{(p)}\right\}_{i=2}^{\infty}$ such that $f_{i}^{(p+1)}(z)=f\left(z_{i}^{(p+1)}+z\right)$, converges uniformly on each compact subset of $D\left(0, r_{p+1}\right)$. Thus, if we consider the subsequence $\left\{z_{i}^{(i)}\right\}_{i=1}^{\infty}$ of $\left\{z_{m}\right\}_{m=1}^{\infty}$, it follows that

$$
f_{i}^{(t)}(z)=f\left(z_{i}^{(t)}+z\right) \text { converges uniformly to a meromorphic }
$$

function $g(z)$ in $Z$ on each compact subset of $Z$.

Here, if we suppose that $g(z)$ is not a constant, we see from a theorem of Hurwitz that $g(z)$ must omit three values on $W$ in $Z$. But, since this does not happen, we can conclude that

$g(z)$ is a constant.

Thus, from (4.1) and (4.2), we see that $f(z)$ is of the second kind in the sense of Yosida, and we obtain a contradiction.

The following Corollary 2 immediately follows from Theorem 3. 
COROLlaRY 2. If $f(z)$ is a meromorphic function in $Z$ of the first $k$ ind in the sense of Yosida, then any ray is a direction of Julia for $f(z)$.

THEOREM 4. Let $f(z)$ be a meromorphic function in $Z$ and $\left\{z_{n}\right\}$, $\left|z_{n}\right| \rightarrow \infty$, be a sequence of points which possesses no subsequence of pseudo- $M^{(0)}$-points for $f(z)$. If

$$
\lim _{n \rightarrow \infty} f\left(z_{n}\right)=w_{0}
$$

where $w_{0}$ is an omitted value of $f(z)$, then $f(z)$ converges uniformly to $w_{0}$ as $|z| \rightarrow \infty$, in the set $\bigcup_{n=1}^{\infty} D\left(z_{n}, r\right)$ for any $r, 0<r<\infty$.

Proof. Suppose that there exists a positive number $r, 0<r<\infty$, such that $f(z)$ does not converge uniformly to $w_{0}$ as $|z| \rightarrow \infty$, in the set $\bigcup_{n=1}^{\infty} D\left(z_{n}, r\right)$. Then, there exist a positive number $\epsilon$, a subsequence $\left\{z_{m}\right\}$ of $\left\{z_{n}\right\}$ and a sequence of points $\left\{z_{m}^{*}\right\},\left|z_{m}^{*}\right| \rightarrow \infty, z_{m}^{*} \in D\left(z_{m}, r\right)$, such that

$$
\chi\left(f\left(z_{m}^{*}\right), w_{0}\right) \geqq \epsilon
$$

where $\chi\left(w_{1}, w_{2}\right)$ denotes the spherical distance between $w_{1}$ and $w_{2}$. Furthermore, by the assumption in Theorem 4 , there exist an increasing sequence of positive numbers $\left\{r_{k}\right\}, r_{k} \uparrow \infty$, and a subsequence $\left\{z_{k}\right\}$ of $\left\{z_{m}\right\}$ such that $f(z)$ omits three values on $W$ in the set $\bigcup_{k=1}^{\infty} D\left(z_{k}, r_{k}\right)$. Hence, by the method analogous to the proof of Theorem 3, we can choose a subsequence $\left\{z_{\imath}^{(t)}\right\}$ of $\left\{z_{k}\right\}$ such that

$$
f_{i}^{(i)}(z)=f\left(z_{i}^{(i)}+z\right) \text { converges uniformly to a meromorphic }
$$

$$
\text { function } g(z) \text { in } Z \text { on each compact subset of } Z \text {. }
$$

Thus, since $f_{i}^{(i)}(0) \rightarrow g(0) \quad(i \rightarrow \infty)$ from $(4.4)$ and $f_{i}^{(i)}(0)=f\left(z_{i}^{(i)}\right) \rightarrow w_{0}$ $(i \rightarrow \infty)$ by the assumption in Theorem 4 , we have

$$
g(0)=w_{0} .
$$

Here, suppose that $g(z)$ is not a constant $w_{0}$. Then, we can conclude from (4.4), (4.5) and a theorem of Hurwitz that each function $f_{i_{l}}^{\left(i_{1}\right)}(z)$ for a subsequence $\left\{i_{l}\right\}$ of $\{i\}$ must take $w_{0}$. But, this contradicts the assumption that $w_{0}$ is an omitted value. Hence, we see that

$$
g(z) \equiv w_{0}
$$

Thus, from (4.4) and (4.6), we have that for $\epsilon$ and $r$ chosen above and for sufficiently large $i$, 


$$
\chi\left(f(z), w_{0}\right)<\epsilon \text { for any } z, z \in D\left(z_{i}^{(i)}, r\right) .
$$

But, this fact (4.7) contradicts (4.3). Therefore, we obtain the conclusion of Theorem 4.

COROLlaRy 3. Let $f(z)$ be an integral function and let $\left\{z_{n}\right\}$, $\left|z_{n}\right| \rightarrow \infty$, be a sequence of points which possesses no subsequence of pseudo- $M^{(0)}$-points for $f(z)$. Then, for each $r, 0<r<\infty$, we have:

(1) If $\lim _{n \rightarrow \infty} f\left(z_{n}\right)=\infty$, then $f(z)$ converges uniformly to infinity as $|z| \rightarrow \infty$, in the set $\bigcup_{n=1}^{\infty} D\left(z_{n}, r\right)$.

(2) If $\left|f\left(z_{n}\right)\right|<K(n=1,2,3, \cdots)$, for some finite constant $K$ which may be dependent on $r$, then $f(z)$ is bounded in the set $\bigcup_{n=1}^{\infty} D\left(z_{n}, r\right)$.

Proof. Only (2) is proved. Suppose that there exists a positive number $r_{0}$ such that $f(z)$ is unbounded in $\bigcup_{n=1}^{\infty} D\left(z_{n}, r_{0}\right)$. Then, there exist a subsequence $\left\{z_{k}\right\}$ of $\left\{z_{n}\right\}$ and a sequence $\left\{z_{k}^{*}\right\}$ of points, satisfying

$$
z_{k}^{*} \in D\left(z_{k}, r_{0}\right) \quad(k=1,2,3, \cdots)
$$

and

$$
\lim _{k \rightarrow \infty} f\left(z_{k}^{*}\right)=\infty
$$

Here, if we suppose that $\left\{z_{k}^{*}\right\}$ is a sequence of pseudo- $M^{(0)}$-points for $f(z)$, we obtain from (4.8) the result that $\left\{z_{k}\right\}$ is also a sequence of pseudo- $M^{(0)}$-points for $f(z)$, which contradicts the assumption in Corollary 3. Hence, $\left\{z_{k}^{*}\right\}$ is not a sequence of pseudo- $M^{(0)}$-points for $f(z)$. Thus, from (4.9) and (1), we obtain the result which contradicts the assumption in (2).

Let $f(z)$ be a meromorphic function in $Z$. A path $L$ tending to $\infty$ is called a pseudo- $M^{(0)}$-path for $f(z)$ if there exists a sequence of pseudo$M^{(0)}$-points for $f(z)$ on $L$.

THEOREM 5. Let $f(z)$ be a meromorphic function in $Z$ and $L$ be an asymptotic path having an asymptotic value $w_{0}$. If $L$ is not a pseudo$M^{(0)}$-path for $f(z)$, then $f(z)$ converges uniformly to $w_{0}$ in the set $\cup_{z \in L}^{\infty} D(z, r)$ for each $r, 0<r<\infty$, where the summation is taken over all $z \in L$.

Proof. We can proceed by the same method as Theorem 4 is proved. Hence, we remark only the following fact.

From the result corresponding to (4.4), we have a sequence $\left\{z_{k}\right\}$, $z_{k} \in L,\left|z_{k}\right| \rightarrow \infty$, and a subsequence $\left\{z_{\imath}^{(i)}\right\}$ of $\left\{z_{k}\right\}$ such that 


$$
f_{i}^{(t)}(z)=f\left(z_{i}^{(i)}+z\right) \text { converges uniformly to a meromorphic }
$$
function $g(z)$ in some neighborhood $\left\{z ;|z| \leqq \epsilon_{0}\right\}$ of $z$ $=0$.

Now, for any $\epsilon, 0<\epsilon \leqq \epsilon_{0}$, and for each $i$, take a point $z_{i}(\epsilon)$ in the nonvoid set $\left\{z ;\left|z-z_{i}^{(i)}\right|=\epsilon\right\} \cap L$, and put $z_{i}(\epsilon)-z_{i}^{(i)}=z_{i}^{*}(\epsilon)$. Let $z^{*}(\epsilon)$ be one of limit points of the set $\bigcup_{i=1}^{\infty} z_{i}^{*}(\epsilon)$ and $\left\{z_{i}^{*}(\epsilon)\right\}_{j=1}^{\infty}$ be the subsequence of $\left\{z^{*}(\epsilon)\right\}$ convergent to $z^{*}(\epsilon)$. Then, from (4.10) we have that

$$
f\left(z_{i},(\epsilon)\right)=f_{i_{j}}^{(i)}\left(z_{i_{j}}^{*}(\epsilon)\right) \rightarrow g\left(z^{*}(\epsilon)\right) \quad(j \rightarrow \infty) .
$$

On the other hand, since $w_{0}$ is an asymptotic value of $f(z)$ along $L$, we have that

$$
f\left(z_{i_{i}}(\epsilon)\right) \rightarrow w_{0} \quad(j \rightarrow \infty) .
$$

Thus, we obtain from (4.11) and (4.12) that for any $\epsilon, 0<\epsilon \leqq \epsilon_{0}$, $g\left(z^{*}(\epsilon)\right)=w_{0}$. Hence, we can conclude that $g(z) \equiv w_{0}$.

Added in Proof. We can easily prove that in Theorem 2

$$
\varlimsup_{r \rightarrow \infty} \frac{T(r, f)}{r^{2}}>0 \quad\left(\text { resp. } \varlimsup_{r \rightarrow \infty} \frac{T(r, f)}{\log ^{2} r}>0\right)
$$

can be replaced by

$$
\varliminf_{r \rightarrow \infty} \frac{T(r, f)}{r^{2}}>0 \quad\left(\text { resp. } \varliminf_{r \rightarrow \infty} \frac{T(r, f)}{\log ^{2} r}>0\right) .
$$

Because, in the proof of Theorem 2 , if for any $r \geqq r_{1}$, we choose a positive integer $n$ satisfying $r_{n+1}>r \geqq r_{n}$, we can obtain the following estimation:

$$
\begin{array}{r}
\frac{T(r, f)}{r^{2}} \geqq \frac{T\left(r_{n}, f\right)}{r_{n+1}^{2}} \geqq \frac{A n(n-1)}{4 \epsilon^{2}(n+1)^{2}} \rightarrow \frac{A}{4 \epsilon^{2}} \\
\left(\text { resp. } \frac{T(r, f)}{\log ^{2} r} \geqq \frac{T\left(r_{n}, f\right)}{\log ^{2} r_{n+1}} \rightarrow A / \log ^{2}\left(\frac{1+\epsilon}{1-\epsilon}\right)\right) .
\end{array}
$$

\section{REFERENCES}

1. V. I. Gavrilov, The behavior of a meromorphic function in the neighborhood of an essentially singular point, Izv. Akad. Nauk SSSR Ser. Math., 30 (1966), 767-788; English transl., Amer. Math. Soc. Translation, 71 (1968), 181-201. 
2. - Some classes of meromorphic functions characterized by their spherical derivative, Izv. Akad. Nauk SSSR Ser. Math., 32 (1968), 735-742; English transl., Math. USSR-Izvestija, 2 (1968), 687-694.

3. O. Lehto and K. I. Virtanen, On the behavior of meromorphic functions in the neighborhood of an isolated singularity, Ann. Acad. Sci. Fenn. Ser. A I 240 (1957), 1-9.

4. S. N. Mergelyan, Uniform approximations to functions of a complex variable, Uspekhi Mat. Nauk (N. S.) 7 (1952), 31-122; English transl., Amer. Math. Soc. Translation, 101 (1954), 1-99.

5. R. Nevanlinna, Eindeutige analytische Funktionen, Springer-Verlag, Berlin-Heidelberg-New York, 1953.

6. K. Noshiro, Contribution to the theory of meromorphic functions in the unit circle, J. Fac. Sci. Hokkaido Univ., 7 (1938), 149-159.

7. A. Ostrowski, Über folgen analytischer funktionen und einige Verschärfungen des Picardschen satzes, Math. Z., 24 (1926), 215-258.

8. K. F. Tse, Some results on value distribution of meromorphic functions in the unit disk, Nagoya Math. J., 34 (1969), 105-119.

9. H. Yoshida, Some remarks on Julia's exceptional functions, (to appear).

10. K. Yosida, On a class of meromorphic functions, Proc. Phys-Math. Soc. Japan, Ser. 3, 16 (1934), 227-235.

Received May 14, 1975 and in revised form December 17, 1975.

Chiba University, JaPAN 




\section{Pacific Journal of Mathematics}

Vol. 64, No. 1

May, 1976

Walter Allegretto, Nonoscillation theory of elliptic equations of order $2 n \ldots \ldots \quad 1$

Bruce Allem Anderson, Sequencings and starters.................. 17

Friedrich-Wilhelm Bauer, A shape theory with singular homology .......... 25

John Kelly Beem, Characterizing Finsler spaces which are

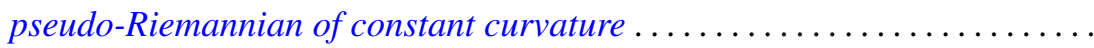

Dennis K. Burke and Ernest A. Michael, On certain point-countable

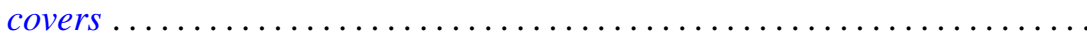

Robert Chen, A generalization of a theorem of Chacon ............... 93

Francis H. Clarke, On the inverse function theorem ................ 97

James Bryan Collier, The dual of a space with the Radon-Nikodým

property ....................................... 103

John E. Cruthirds, Infinite Galois theory for commutative rings ............ 107

Artatrana Dash, Joint essential spectra......................... 119

Robert M. DeVos, Subsequences and rearrangements of sequences in FK

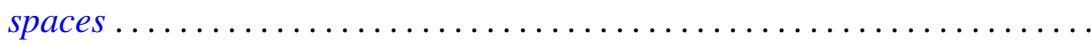

Geoffrey Fox and Pedro Morales, Non-Hausdorff multifunction generalization

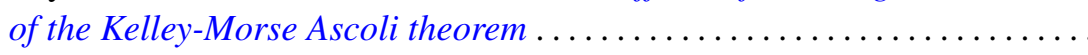

Richard Joseph Fleming, Jerome A. Goldstein and James E. Jamison, One

parameter groups of isometries on certain Banach spaces.............

Robert David Gulliver, II, Finiteness of the ramified set for branched

immersions of surfaces

Kenneth Hardy and István Juhász, Normality and the weak cb property ...... 167

C. A. Hayes, Derivation of the integrals of $L^{(q)}$-functions.

Frederic Timothy Howard, Roots of the Euler polynomials .

Robert Edward Jamison, II, Richard O'Brien and Peter Drummond Taylor, On

embedding a compact convex set into a locally convex topological vector space ....................................

Andrew Lelek, An example of a simple triod with surjective span smaller than span ...

Janet E. Mills, Certain congruences on orthodox semigroups

Donald J. Newman and A. R. Reddy, Rational approximation of $e^{-x}$ on the positive real axis.

John Robert Quine, Jr., Homotopies and intersection sequences ...

Nambury Sitarama Raju, Periodic Jacobi-Perron algorithms and fundamental units ....

Herbert Silverman, Convexity theorems for subclasses of univalent functions. . .

Charles Frederick Wells, Centralizers of transitive semigroup actions and endomorphisms of trees.........................

Volker Wrobel, Spectral approximation theorems in locally convex spaces ..................... 